\title{
A Classical Approach of Unified Field
}

\author{
Mukul Chandra Das ${ }^{1,2, *}$, Rampada Misra ${ }^{3}$ \\ ${ }^{1}$ Singhania University, Pacheri Bari - 333515, Jhunjhunu, Rajasthan, India \\ ${ }^{2}$ Satmile High School, Satmile - 721452, Contai, West Bengal, India \\ ${ }^{3}$ Department of Electronics, Vidyasagar University, Midnapore - 721102, West Bengal, India \\ *Email address: mukuldas.100@gmail.com
}

\begin{abstract}
Photons contribute electromagnetic field that accompanies the gravitational field. These two fields may be, mutually, transformed through a kind of transformation matrix dependent upon the boundary conditions of the system. In this work trial would be made to find the nature of this transformation matrix considering a super system of photon in which both fields are unified. The unification of electromagnetic and gravitational fields leads to the concept of fundamental charge.
\end{abstract}

Keywords: Electromagnetic field; gravitational field; photon; unified field

\section{INTRODUCTION}

A system may be described by two or more frames of references. Space-time coordinates of an event could be transformed from one frame to another using a proper transformation matrix. So, it would be required to formulate the transformation matrix for a system where inertial and non-inertial frames are present. This would be applied to system of photons where both of the frames may, assumed, to be present due to their angular momentum along propagation. This leads to unification of fields related to the motion of photons which, again, gives a concept of fundamental charge.

The unified field theory accepts a unification of four known fields. Unification of strong, electromagnetic and weak field has been included in grand unified theory but, gravity has not been included in this scheme in a satisfactory manner although, Einstein himself [18], Schrodinger [19], Maxwell [20] tried to unify within a frame associating electromagnetism with a new geometry of space-time. Einstein [1] suggested the field equation for the gravity of an electro-magnetic wave as $G_{\alpha \beta}=-K T(E)_{a b}$ where, $G_{\alpha \beta}$ is the Einstein tensor, and $K$ is the coupling constant. After a long year from Einstein, the weak force and the electromagnetic force were unified in a theory presented independently by L. Glashow [21], A. Salam [22], S. Weinberg [23]. But, gravity is developing independently and unification of it with other fields has not been solved until now in a satisfactory manner.

On the other hand, prediction of general relativity is that light is also a source of gravity. In this respect, many year ago, Tolmar [24] observed that light may be more effective than matter in generating a gravitational field. Again, photon carries electromagnetic field 
accompanying the gravitational field [2-8]. On the basis of the present concept of photon, present work tries to relate electromagnetic field and gravitation field of photon in its system using a transformation matrix.

\section{CONCEPT OF L-L, S-S, S-L SYSTEMS}

A particle may possess two simultaneous superimposed velocities as well as spins. These types of superimposition may be classified as discussed below.

\section{1. L-L System}

In $[9,17]$ it is stated that frames $S$ and $S_{1}$ have both their co-ordinate axes aligned and $S_{1}$ is moving at a velocity $u$ along $X_{1}$ axis as observed by $S$. The inertial frame $S_{1}$ has another co-ordinate reference frame $S_{2}$ where $X_{2}$ axis of $S_{2}$ is rotated by an angle $\theta$ counter clockwise with respect to $S_{1}$ on $X_{1} Y_{1}$ plane. Frames $S_{2}$ and $S_{3}$ have both their co-ordinate axes aligned and $S_{3}$ is moving at a velocity $v$ along $X_{3}$ axis as observed by $S_{2}$. Then magnitude of resultant velocity of an event of $S_{3}$ as observed by $S$ would be

$$
w=\sqrt{\frac{u^{2}+v^{2}+2 u v \cos \theta-\left(u^{2} v^{2} \sin ^{2} \theta\right) / c^{2}}{(u v \cos \theta) / c^{2}+1}}
$$

There are two linear (L) velocities which have been composed in (1) and they interact with each other. So, it is the L-L interaction and the system is the L-L system.

According to $[9,17]$, coordinate transformation matrix from $S$ to $S_{3}$ would be

$$
\begin{gathered}
N_{i j}=L_{x v} R_{x y(\theta)} L_{x u}= \\
\left(\begin{array}{cccc}
\gamma_{u} \gamma_{v}\left(\frac{u v}{c^{2}}+\cos \theta\right) & \gamma_{v} \sin \theta & 0 & -\gamma_{u} \gamma_{v}(v+u \cos \theta) \\
-\gamma_{u} \sin \theta & \cos \theta & 0 & \gamma_{u} u \sin \theta \\
0 & 0 & 1 & 0 \\
\frac{-\gamma_{u} \gamma_{v}}{c^{2}}(v \cos \theta+u) & -\frac{\gamma_{v} \sin \theta}{c^{2}} & 0 & \gamma_{u} \gamma_{v}\left(\frac{u v \cos \theta}{c^{2}}+1\right)
\end{array}\right)
\end{gathered}
$$

where, 


$$
L_{x u}=\left(\begin{array}{cccc}
\gamma_{u} & 0 & 0 & -u \gamma_{u} \\
0 & 1 & 0 & 0 \\
0 & 0 & 1 & 0 \\
\frac{-u \gamma_{u}}{c^{2}} & 0 & 0 & \gamma_{u}
\end{array}\right), R_{x y(\theta)}=\left(\begin{array}{cccc}
\cos \theta & \sin \theta & 0 & 0 \\
-\sin \theta & \cos \theta & 0 & 0 \\
0 & 0 & 1 & 0 \\
0 & 0 & 0 & 1
\end{array}\right), L_{x v}=\left(\begin{array}{cccc}
\gamma_{v} & 0 & 0 & -v \gamma_{v} \\
0 & 1 & 0 & 0 \\
0 & 0 & 1 & 0 \\
\frac{-v \gamma_{v}}{c^{2}} & 0 & 0 & \gamma_{v}
\end{array}\right)
$$

The matrix for coordinate transformation from $S_{3}$ to $S$ would be

$$
\begin{gathered}
\bar{N}_{i j}=L_{x(-u)} R_{x y(-\theta)} L_{x(-v)} \\
=\left(\begin{array}{cccc}
\gamma_{u} \gamma_{v}\left(\frac{u v}{c^{2}}+\cos \theta\right) & -\gamma_{u} \sin \theta & 0 & \gamma_{u} \gamma_{v}(v \cos \theta+u) \\
\gamma_{v} \sin \theta & \cos \theta & 0 & \gamma_{v} v \sin \theta \\
0 & 0 & 1 & 0 \\
\frac{\gamma_{u} \gamma_{v}}{c^{2}}(v+u \cos \theta) & \frac{-\gamma_{u} u \sin \theta}{c^{2}} & 0 & \gamma_{u} \gamma_{v}\left(\frac{u v \cos \theta}{c^{2}}+1\right)
\end{array}\right)
\end{gathered}
$$

Using $\bar{N}_{i j}$ we get the relation for coordinate transformation from $S_{3}$ to $S$ as

$$
X(x, y, z, t)=\bar{N}_{i j} X^{\prime}\left(x^{\prime}, y^{\prime}, z^{\prime}, t^{\prime}\right)
$$

Here, $X(x, y, z, t)=\left(\begin{array}{c}x \\ y \\ z \\ t\end{array}\right)$ and $X^{\prime}\left(x^{\prime}, y^{\prime}, z^{\prime}, t^{\prime}\right)=\left(\begin{array}{c}x^{\prime} \\ y^{\prime} \\ z^{\prime} \\ t^{\prime}\end{array}\right)$

Similarly, using $N_{i j}$, the relation for coordinate transformation from $S$ to $S_{3}$ would be

$$
X^{\prime}\left(x^{\prime}, y^{\prime}, z^{\prime}, t^{\prime}\right)=N_{i j} X(x, y, z, t)
$$

Now let, $\varphi^{\prime}\left(r^{\prime}, t^{\prime}\right)$ be a field function of prime frame $\left(S_{3}\right)$, then it would appear as $\varphi(r, t)$ to the view of an observer of rest frame $(S)$. Using $\bar{N}_{i j}$ in (3) and following (4), we may write a relation between $\varphi(r, t)$ and $\varphi^{\prime}\left(r^{\prime}, t^{\prime}\right)$ as

$$
\varphi_{\alpha}(r, t)=\Lambda \bar{N}_{i j} \varphi_{\alpha}^{\prime}\left(r^{\prime}, t^{\prime}\right)
$$


where, $\quad \varphi_{\alpha}(r, t)=\left(\begin{array}{c}\varphi_{x} \\ \varphi_{y} \\ \varphi_{z} \\ \varphi_{t}\end{array}\right)$ and $\varphi_{\alpha}^{\prime}\left(r^{\prime}, t^{\prime}\right)=\left(\begin{array}{c}\varphi_{x}^{\prime} \\ \varphi_{y}^{\prime} \\ \varphi_{z}^{\prime} \\ \varphi_{t}^{\prime}\end{array}\right)$

Again using $N_{i j}$ in (2) and following (5), we may write the relation as

$$
\varphi_{\alpha}^{\prime}\left(r^{\prime}, t^{\prime}\right)=\bar{\Lambda} N_{i j} \varphi_{\alpha}(r, t)
$$

where, $\Lambda$ and $\bar{\Lambda}$ would be the matrices having constant element depending upon the systems.

\section{2. S-S System}

A particle may possess two or more simultaneous superimposed spins. For clarity of two simultaneous superimposed spins (Spin-Spin interaction), it is stated that frames $S$ and $S_{1}$ have both their $X$ axes aligned and $S_{1}$ is moving at an angular velocity $\omega_{1}$ about $X_{1}$ axis as observed by $S$. The frame $S_{1}$ has another co-ordinate reference frame $S_{2}$, where $X_{2}$ axis of $S_{2}$, are rotated by an angle $\theta$ counter clockwise with respect to $S_{1}$ on $X_{1} Y_{1}$ plane. Frames $S_{2}$ and $S_{3}$ have both their $X$ axes aligned and $S_{3}$ is moving at an angular velocity $\omega_{2}$ about $X_{3}$ axis as observed by $S_{2}$. For the case when the origin of frames are same with respect to $S$ and the particle be at the origin of $S_{3}$, then it possesses Spin-Spin (S-S) interaction with respect to frame $S$. The system would be denoted by the term S-S system and coordinate transformation matrix from $S$ to $S_{3}$ would be $S_{i j}$ also this would be the matrix product

$R_{y z\left(\omega_{2} t\right)} R_{x y(\theta)} R_{y z\left(\omega_{1} t\right)}$ where,

$R_{y z\left(\omega_{i} t\right)}=\left(\begin{array}{cccc}1 & 0 & 0 & 0 \\ 0 & \cos \omega_{i} t & \sin \omega_{i} t & 0 \\ 0 & -\sin \omega_{i} t & \cos \omega_{i} t & 0 \\ 0 & 0 & 0 & 1\end{array}\right), i=1,2$ and $R_{x y(\theta)}$ is as specified in section-2.1.

Hence, we get the relations between co-ordinates of different frames $S, S_{1}, S_{2}$ and $S_{3}$ as stated below

$$
\left(\begin{array}{l}
x_{1} \\
y_{1} \\
z_{1} \\
t_{1}
\end{array}\right)=\left(\begin{array}{cccc}
1 & 0 & 0 & 0 \\
0 & \cos \omega_{1} t & \sin \omega_{1} t & 0 \\
0 & -\sin \omega_{1} t & \cos \omega_{1} t & 0 \\
0 & 0 & 0 & 1
\end{array}\right)\left(\begin{array}{l}
x \\
y \\
z \\
t
\end{array}\right), \quad\left(\begin{array}{l}
x_{2} \\
y_{2} \\
z_{2} \\
t_{2}
\end{array}\right)=\left(\begin{array}{cccc}
\cos \theta & \sin \theta & 0 & 0 \\
-\sin \theta & \cos \theta & 0 & 0 \\
0 & 0 & 1 & 0 \\
0 & 0 & 0 & 1
\end{array}\right)\left(\begin{array}{l}
x_{1} \\
y_{1} \\
z_{1} \\
t_{1}
\end{array}\right) \text { and }
$$




$$
\left(\begin{array}{l}
x_{3} \\
y_{3} \\
z_{3} \\
t_{3}
\end{array}\right)=\left(\begin{array}{cccc}
1 & 0 & 0 & 0 \\
0 & \cos \omega_{2} t & \sin \omega_{2} t & 0 \\
0 & -\sin \omega_{2} t & \cos \omega_{2} t & 0 \\
0 & 0 & 0 & 1
\end{array}\right)\left(\begin{array}{l}
x_{2} \\
y_{2} \\
z_{2} \\
t_{2}
\end{array}\right)
$$

Therefore, the transformation matrix for coordinates of an event from $S$ to $S_{3}$ as shown below

$$
S_{i j}=R_{y z\left(\omega_{2} t\right)} R_{x y(\theta)} R_{y z\left(\omega_{1} t\right)}
$$

$$
=\left(\begin{array}{ccccc}
1 & 0 & 0 & 0 \\
0 & \cos \omega_{2} t & \sin \omega_{2} t & 0 \\
0 & -\sin \omega_{2} t & \cos \omega_{2} t & 0 \\
0 & 0 & 0 & 1
\end{array}\right)\left(\begin{array}{cccc}
\cos \theta & \sin \theta & 0 & 0 \\
-\sin \theta & \cos \theta & 0 & 0 \\
0 & 0 & 1 & 0 \\
0 & 0 & 0 & 1
\end{array}\right)\left(\begin{array}{cccc}
1 & 0 & 0 & 0 \\
0 & \cos \omega_{1} t & \sin \omega_{1} t & 0 \\
0 & -\sin \omega_{1} t & \cos \omega_{1} t & 0 \\
0 & 0 & 0 & 1
\end{array}\right)
$$

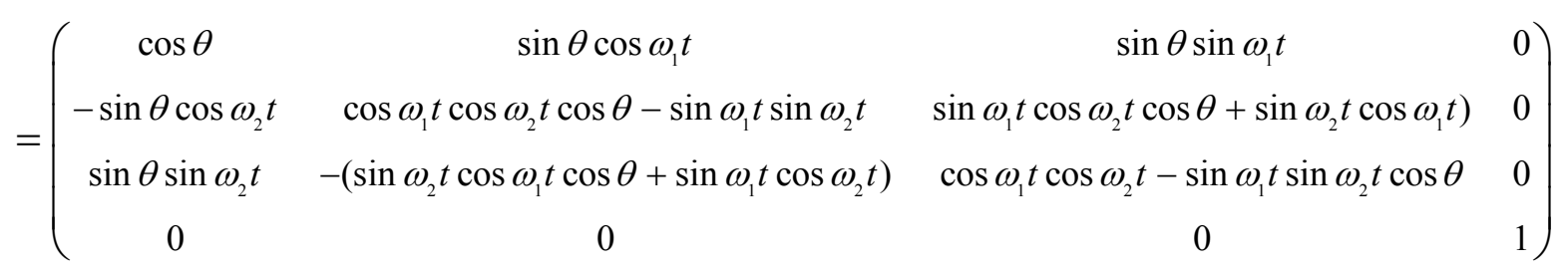

Similarly the matrix for coordinate transformation from $S_{3}$ to $S$ would be

$$
\begin{gathered}
\bar{S}_{i j}=R_{y z\left(-\omega_{1} t\right)} R_{x y(-\theta)} R_{y z\left(-\omega_{2} t\right)} \\
=\left(\begin{array}{cccc}
1 & 0 & 0 & 0 \\
0 & \cos \omega_{1} t & -\sin \omega_{1} t & 0 \\
0 & \sin \omega_{1} t & \cos \omega_{1} t & 0 \\
0 & 0 & 0 & 1
\end{array}\right)\left(\begin{array}{cccc}
\cos \theta & -\sin \theta & 0 & 0 \\
\sin \theta & \cos \theta & 0 & 0 \\
0 & 0 & 1 & 0 \\
0 & 0 & 0 & 1
\end{array}\right)\left(\begin{array}{cccc}
1 & 0 & 0 & 0 \\
0 & \cos \omega_{2} t & -\sin \omega_{2} t & 0 \\
0 & \sin \omega_{2} t & \cos \omega_{2} t & 0 \\
0 & 0 & 0 & 1
\end{array}\right)
\end{gathered}
$$

$=\left(\begin{array}{cccc}\cos \theta & -\sin \theta \cos \omega_{2} t & \sin \theta \sin \omega_{2} t & 0 \\ \sin \theta \cos \omega_{1} t & \cos \omega_{1} t \cos \omega_{2} t \cos \theta-\sin \omega_{1} t \sin \omega_{2} t & -\left(\sin \omega_{2} t \cos \omega_{1} t \cos \theta+\sin \omega_{1} t \cos \omega_{2} t\right) & 0 \\ \sin \theta \sin \omega_{1} t & \sin \omega_{1} t \cos \omega_{2} t \cos \theta+\sin \omega_{2} t \cos \omega_{1} t & \cos \omega_{1} t \cos \omega_{2} t-\sin \omega_{1} t \sin \omega_{2} t \cos \theta & 0 \\ 0 & 0 & 0 & 1\end{array}\right)$ 
This leads to the relation for coordinate transformation from $S_{3}$ to $S$ in this system as

$$
X(x, y, z, t)=\bar{S}_{i j} X^{\prime}\left(x^{\prime}, y^{\prime}, z^{\prime}, t^{\prime}\right)
$$

\section{3. S-L System}

For clarity of another interaction specified as Spin-Linear (S-L) interaction, it is stated by the same way that frames $S$ and $S_{1}$ have both their $X$ axes aligned and $S_{1}$ is moving at an angular velocity $\omega$ about $X_{1}$ axis as observed by $S$. The frame $S_{1}$ has another co-ordinate reference frame $S_{2}$, where $X_{2}$ axis of $S_{2}$, are rotated by an angle $\theta$ counter clockwise with respect to $S_{1}$ on $X_{1} Y_{1}$ plane. Frames $S_{2}$ and $S_{3}$ have both their $X$ axes aligned and $S_{3}$ is moving at a linear velocity $v$ along $X_{3}$ axis as observed by $S_{2}$. For this case, if the particle be at the origin of $S_{3}$, then it possesses Spin-Linear (S-L) interaction with respect to frame $S$. The system would be denoted by the term S-L system and coordinate transformation matrix from $S$ to $S_{3}$ would be $Z_{i j}$ which would be the matrix product $L_{x v} R_{x y(\theta)} R_{y z(\omega t)}$. Now, using the matrices $L_{x v}, \quad R_{x y(\theta)}$ and $R_{y z(\omega t)}$ as specified in section-2.1 and 2.2 we get the co-ordinate transformation matrix as given below

$$
\begin{aligned}
Z_{i j} & =L_{x v} R_{x y(\theta)} R_{y z(\omega t)} \\
& =\left(\begin{array}{cccc}
\gamma_{v} & 0 & 0 & -v \gamma_{v} \\
0 & 1 & 0 & 0 \\
0 & 0 & 1 & 0 \\
\frac{-v \gamma_{v}}{c^{2}} & 0 & 0 & \gamma_{v}
\end{array}\right)\left(\begin{array}{cccc}
\cos \theta & \sin \theta & 0 & 0 \\
-\sin \theta & \cos \theta & 0 & 0 \\
0 & 0 & 1 & 0 \\
0 & 0 & 0 & 1
\end{array}\right)\left(\begin{array}{cccc}
1 & 0 & 0 & 0 \\
0 & \cos \omega t & \sin \omega t & 0 \\
0 & -\sin \omega t & \cos \omega t & 0 \\
0 & 0 & 0 & 1
\end{array}\right) \\
& =\left(\begin{array}{cccc}
\gamma \cos \theta & \gamma \sin \theta \cos \omega t & \gamma \sin \theta \sin \omega t & -v \gamma \\
-\sin \theta & \cos \theta \cos \omega t & \cos \theta \sin \omega t & 0 \\
0 & -\sin \omega t & \cos \omega t & 0 \\
\frac{-v \gamma \cos \theta}{c^{2}} & \frac{-v \gamma \sin \theta \cos \omega t}{c^{2}} & \frac{-v \gamma \sin \theta \sin \omega t}{c^{2}} & \gamma
\end{array}\right)
\end{aligned}
$$

Similarly the matrix for coordinate transformation from $S_{3}$ to $S$ would be

$$
\bar{Z}_{i j}=R_{y z(-\omega t)} R_{x y(-\theta)} L_{(-x v)}
$$




$$
\begin{aligned}
& =\left(\begin{array}{cccc}
1 & 0 & 0 & 0 \\
0 & \cos \omega t & -\sin \omega t & 0 \\
0 & \sin \omega t & \cos \omega t & 0 \\
0 & 0 & 0 & 1
\end{array}\right)\left(\begin{array}{cccc}
\cos \theta & -\sin \theta & 0 & 0 \\
\sin \theta & \cos \theta & 0 & 0 \\
0 & 0 & 1 & 0 \\
0 & 0 & 0 & 1
\end{array}\right)\left(\begin{array}{cccc}
\gamma_{v} & 0 & 0 & v \gamma_{v} \\
0 & 1 & 0 & 0 \\
0 & 0 & 1 & 0 \\
\frac{v \gamma_{v}}{c^{2}} & 0 & 0 & \gamma_{v}
\end{array}\right) \\
& =\left(\begin{array}{cccc}
\gamma \cos \theta & -\sin \theta & 0 & \gamma v \cos \theta \\
\gamma \sin \theta \cos \omega t & \cos \theta \cos \omega t & -\sin \omega t & v \gamma \sin \theta \cos \omega t \\
\gamma \sin \theta \sin \omega t & \cos \theta \sin \omega t & \cos \omega t & v \gamma \sin \theta \sin \omega t \\
\frac{\gamma v}{c^{2}} & 0 & 0 & \gamma
\end{array}\right)
\end{aligned}
$$

This leads to the relation for coordinate transformation from $S_{3}$ to $S$ in this systems (S-L) as

$$
X(x, y, z, t)=\bar{Z}_{i j} X^{\prime}\left(x^{\prime}, y^{\prime}, z^{\prime}, t^{\prime}\right)
$$

\section{VELOCITY AND SYSTEM OF PHOTON}

Velocity of Photon: In [10-13] it is implied that light is an electromagnetic wave. It carries electric and magnetic fields. But photon is a particle whose kinetic energy is $h v$, having mass $h v / c^{2}$. Photon has spin angular momentum $J= \pm \hbar$ about an axis or along the direction of propagation. According to [13] photon may be considered as a small mass $\left(m=h v / c^{2}\right)$ concentrated in a ring of radius $r$ and rotates at velocity of light $(u=c)$ and it also has linear motion with velocity of light along the axis of rotation $(v=c)$. Therefore, there are two velocities (in a composed form like S-L system) present in the system of photon which may be of two types: right rotating photon and left rotating photon [12].

Super System of Photon (SSP): To make the above concept of photon more clear, let, photon be not only a particle or a wave but it is a system and a kind of S-L interaction is happening in this system. Superimposed velocities of sub-photonic particle (as in Sec-4) in this system would be $u(=\omega r)=v=c, \omega=2 \pi v$ where, $v$ is the frequency of the system. This system could be denoted by the term 'Super System of Photon' (SSP). To clarify the SSP, we can consider four reference frames $S, S_{1}, S_{2}$ and $S_{3}$ as discussed in section-2.3 where, $S$ is the rest or observer's frame and the other three reference frames $\left(S_{1}, S_{2}\right.$ and $\left.S_{3}\right)$ are in the SSP which are also movable as in section-2.3. Therefore, transformation matrix in this system would be the form of $Z_{i j}$ and $\bar{Z}_{i j}$ which are respectively specified in (10) and (11). But, the system carries totally kinetic energy; there is no rest mass in this system and $\gamma$ would not be 
exactly same as that defined in Lorentz transformation matrix. Therefore, in the picture of SSP, $Z_{i j}$ and $\bar{Z}_{i j}$ would be the special matrices in which

a) $\gamma$ would be the ratio of energy density as in equation (14A) and (14B)

b) velocity $v$ is the speed of light $(v=c)$ and $\omega=2 \pi v$

\section{RELATION BETWEEN E.M. FIELD AND GRAVITATIONAL FIELD OF PHOTON}

Since photons contribute to the stress-energy tensor, they exert a gravitational attraction on other objects, according to the theory of general relativity. Several works [2-8] imply that photon contains electromagnetic field accompanying the gravitational field; it can interact with gravitational field; source of relativistic mass of photon is gravitational interaction; also, photon may decay into one or more graviton. Again photon is a system which carries electromagnetic field, origin of which should be gravitational interaction. This means that the origin of the system (photon) is gravitons which may be called sub-photonic particles

Above discussions imply that photon has electro-magnetic field accompanying the gravitational field. In SSP electromagnetic field and gravitational field are unified due to the fact that in SSP gravitational interaction is happening and results in the electro-magnetic field with respect to an observer. This means that, the system transforms the gravitational field to the electro-magnetic field with respect to an observer.

To make transformation from gravitational field to electro-magnetic field in SSP let, gravitational field function in the frame $S_{3}$ be $G^{\prime}\left(r^{\prime}, t^{\prime}\right)$ which would be electromagnetic field function $\psi(r, t)=\psi\{E(r, t), B(r, t)\}$ as observed by $S$.

Following (6) and using $\bar{Z}_{i j}$ matrix as specified in (11) we can consider a relation between $G^{\prime}\left(r^{\prime}, t^{\prime}\right)$ and $\psi(r, t)$ as

$$
\psi_{\alpha}(r, t)=\Upsilon_{1} \bar{Z}_{i j} G_{\alpha}^{\prime}\left(r^{\prime}, t^{\prime}\right)
$$

where, $\Upsilon_{1}$ is matrix having constant element depending upon the system. Here, $\bar{Z}_{i j}$ is canonical transformation matrix in SSP as discussed in section-3. This matrix would be of the form (11). Again, $\gamma$ in the transformation matrix would be the function of energy density of the system. To clarify it let, gravitational energy density and electromagnetic energy density respectively be $\rho_{g}$ and $\rho_{e . m}$ in the system of photon. Then we can write

$$
\gamma=\rho_{e . m .} / \rho_{g}
$$

This may be written as

$$
\gamma=\left|\frac{\delta \psi(r, t)}{\delta t}\right| /\left|\frac{\delta G^{\prime}\left(r^{\prime}, t^{\prime}\right)}{\delta t}\right|
$$


where, respectively $\psi(r, t)$ and $G^{\prime}\left(r^{\prime}, t^{\prime}\right)$ are considered as the wave function of electromagnetic field and gravitational field in the system of it (photon). Using $Z_{i j}$ in (10) we get from (13)

$$
G_{\alpha}^{\prime}\left(r^{\prime}, t^{\prime}\right)=\Upsilon_{2} Z_{i j} \psi_{\alpha}(r, t)
$$

where, $\psi_{\alpha}(r, t)=\left(\begin{array}{l}\psi_{x} \\ \psi_{y} \\ \psi_{z} \\ \psi_{t}\end{array}\right)$ and $G_{\alpha}^{\prime}\left(r^{\prime}, t^{\prime}\right)=\left(\begin{array}{c}G_{x}^{\prime} \\ G_{y}^{\prime} \\ G_{z}^{\prime} \\ G_{t}^{\prime}\end{array}\right)$

Following the same convention, we may assume a relation between two fields $\psi^{\prime}\left(r^{\prime}, t^{\prime}\right)$ and $G(r, t)$ as

$$
G_{\alpha}(r, t)=\Upsilon_{3} \bar{Z}_{i j} \psi_{\alpha}^{\prime}\left(r^{\prime}, t^{\prime}\right)
$$

where, $\psi^{\prime}\left(r^{\prime}, t^{\prime}\right)$ would be the electromagnetic field function, $\Upsilon_{2}$ and $\Upsilon_{3}$ are matrices like $\Upsilon_{1}$ while $G(r, t$, may be one kind of gravitational field which is created by electromagnetic interaction in S-L system and $\gamma=\left|\frac{\delta G(r, t)}{\delta t}\right| /\left|\frac{\delta \psi^{\prime}\left(r^{\prime}, t^{\prime}\right)}{\delta t}\right|$

However, gravitational field and electromagnetic field are unified in SSP by the relations in (13) and (14).

Above ideas may be extended to Einstein's field equation as proposed below Now, we recall that the original Einstein's equation for gravitation [25] is:

$$
R_{\mu \nu}-\frac{1}{2} g_{\mu v} R+g_{\mu v} \Lambda=-\frac{8 \pi G}{c^{4}} T_{(g) \mu v}
$$

where, $T_{(g) \mu v}$ be the energy- momentum tensor of gravitational $(g)$ field.

On the other hand energy-momentum tensor of electro-magnetic $(\mathrm{em})$ field [26] is

$$
T_{(e m)}^{\mu \nu}=\frac{1}{\mu_{0}}\left(F^{\mu \alpha} F_{\alpha}^{v}-\frac{1}{4} \eta^{\mu v} F_{\alpha \beta} F^{\alpha \beta}\right)
$$

where, $F_{\alpha \beta}$ is the electro-magnetic field tensor. 
Since field is the function of energy-momentum, so, following (13) we can write the relations between gravitational energy-momentum tensor $\left(T_{(g) \mu v}\right)$ and electromagnetic energymomentum tensor $\left(T_{(e m)}^{\mu v}\right)$ in the system of photon as

$$
T_{(e m)}^{\mu v}=\bar{\zeta} \bar{Z}_{i j} T_{(g) \mu v}
$$

Similarly, following (14) we can write

$$
T_{(g) \mu v}=\zeta Z_{i j} T_{(e m)}^{\mu v}
$$

where, $\zeta$ and $\bar{\zeta}$ would be coupling constants

Now, using (19) and (17), we get from (16) the equation

$$
R_{\mu \nu}-\frac{1}{2} g_{\mu \nu} R+g_{\mu \nu} \Lambda=-\frac{8 \pi G}{c^{4}} \zeta Z_{i j} T_{(e m)}^{\mu v}=-\frac{8 \pi G}{c^{4} \mu_{0}} \zeta Z_{i j}\left(F^{\mu \alpha} F_{\alpha}^{v}-\frac{1}{4} \eta^{\mu v} F_{\alpha \beta} F^{\alpha \beta}\right)
$$

Similarly, following (17), (18) and (16), we can write

$$
F^{\mu \alpha} F_{\alpha}^{v}-\frac{1}{4} \eta^{\mu v} F_{\alpha \beta} F^{\alpha \beta}=\mu_{0} T_{(e m)}^{\mu v}=\mu_{0} \bar{\zeta} \bar{Z}_{i j} T_{(g) \mu v}=\frac{\mu_{0} c^{4}}{8 \pi G} \bar{\zeta} \bar{Z}_{i j}\left(R_{\mu v}-\frac{1}{2} g_{\mu v} R+g_{\mu v} \Lambda\right)
$$

Therefore, in the system of photon, electromagnetism and gravitation would be related by equations (20) and (21) which are also connected with Einstein's field equation

\section{CONCEPT OF FUNDAMENTAL CHARGE}

a) Photons may be of two types [10-13]: right rotating photon and left rotating photon. These two types of photons imply the two types of S-L interaction in it. This means that two types of energy momentum nature (which is basis of field) exist in photons.

b) From [14-16] we get a concept that photon charge (positive and negative) exists but it has an upper limit $\left(<3 \times 10^{-35} e\right)$. Each types of charge would be related to the type of energy-momentum nature hence, on the type of S-L interactions in it.

c) From [4,5] we get, source of relativistic mass of photon is due to gravitational interactions within it, photon can decay into one or more graviton. Also, Einstein's prediction as well as consideration is that all fields are rooted in gravity. 
d) According to the discussions in Sec-3 and Sec-4, a photon is not only a particle or wave but a SSP in which gravitational interaction is happening resulting in electromagnetic field with respect to an observer. Now, the system (SSP) is called charged (since photon contains charge). This indicates that the system (SSP) contains charge the origin of which is gravitons (due to the fact that electro-magnetic field is created by gravitational interaction in SSP)

Therefore, charged quanta is a fundamental system (like photon) in which S-L (or may be S-S) interaction as well as gravitational interaction should be present.

\section{CONCLUSIONS}

A particle or a system of particles may possess two simultaneous superimposed velocities. There may be L-L, S-S, S-L interactions which would give us different effective velocities for different type of interactions. Considering transformation matrix in the system we may transform a type of field to another type with respect to frame of reference. For a particle existing in systems like SSP, its gravitational field and electromagnetic field may be interrelated. Then the system or particle may acquire fundamental charge. Therefore, charged quanta is a fundamental system (like photon) in which S-L (or may be S-S) interaction as well as gravitational interaction should be present.

\section{References}

[1] Einstein A., Physics and relativity (1936) in Ideas and Opinions, Crown, New York 1954, p. 311.

[2] H. Stephani, J. Stewart, General Relativity: An Introduction to the Theory of Gravitational Field, Cambridge University Press 1990, p. 86 ff, 108 ff.

[3] W. B. Bonnor, General Relativity and Gravitation 41 (2009) 77-85.

[4] M. A. Grado-Caffaro, M. Grado-Caffaro, Optik-International Journal of Light and Electron Optics 123 (2012) 814-815.

[5] D. F. Crawford, Nature 254 (1975) 313-314.

[6] A. Accioly, R. Paszko, Physical Review D 69 (2004) 107501.

[7] J. T. Mendonca, L. O’C. Drury, Physical Review-D 65 (2001) 024026.

[8] S. C. İnan, Chinese Physics Letter 29 (2012) 031301.

[9] C. Iyer, G. M. Prabhu, Journal of Physical and Natural Sciences 1(2) (2007) 1-8.

[10] C. V. Raman, S. Bhagavantam, Indian Journal of Physics 6 (1931) 353-366.

[11] Martin Harwit, The Astrophysical Journal 597 (2003) 1266-1270.

[12] D. Zu, Progress in Electromagnetics Research Letters 1 (2008) 109-118. 
[13] Y. Rocard, Thermodynamique, Masson, Paris 1957, p. 250.

[14] C. Amsler, Particle Data Group, Physics Letters B 667 (2008) 1-6.

[15] V. V. Kobychev, S. B. Porov, Astronomy Letters 31 (2005) 147-151.

[16] C. Sivaram, Bulletin of the Astronomical Society of India 27 (1999) 627-630.

[17] M. C. Das, R. Misra, International Journal of Astronomy and Astrophysics 2(2) (2012) 97-100.

[18] A. Einstein, Geometry and Experience Sidelights on Relativity, Denver 1922.

[19] L. Bass, E. Schrodinger, Proc. Roy. Soc. A, (1955) 232.

[20] J. C. Maxwell, A Treatise on Electricity and magnetism, Dover 1954.

[21] L. Glashow, Reviews of Modern Physics 52 (1980) 539-543.

[22] A. Salam, Reviews of Modern Physics 52 (1980) 525-538.

[23] S. Weinberg, Re-views of Modern Physics 52 (1980) 515- 523.

[24] R. C. Tolmar, Relativity, Thermodynamics and Cosmology, Oxford University Press, Oxford 1934, p.272.

[25] A. Einstein, Annalen der Physik 354(7) (1916) 769-822, doi:10.1002/andp.19163540702.

[26] J. A. Wheeler, C. Misner, K. S. Thorne, Gravitation, W. H. Freeman \& Co, 1973. 